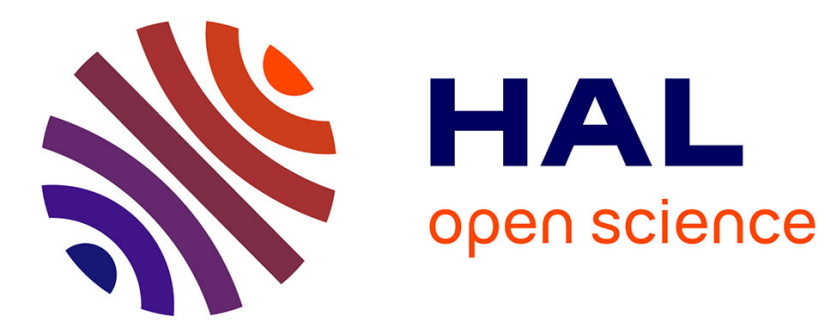

\title{
Accélérateurs et nouveaux concepts d'accélération de particules
}

B. Heusch, G. Matthieussent

\section{To cite this version:}

B. Heusch, G. Matthieussent. Accélérateurs et nouveaux concepts d'accélération de particules. Accelerateurs Et Nouveaux Concepts D'Acceleration De Particules, 1987, Strasbourg, France. pp.1417-1419, 10.1051/rphysap:019880023090141700 . jpa-00245963

\section{HAL Id: jpa-00245963 https://hal.science/jpa-00245963}

Submitted on 1 Jan 1988

HAL is a multi-disciplinary open access archive for the deposit and dissemination of scientific research documents, whether they are published or not. The documents may come from teaching and research institutions in France or abroad, or from public or private research centers.
L'archive ouverte pluridisciplinaire HAL, est destinée au dépôt et à la diffusion de documents scientifiques de niveau recherche, publiés ou non, émanant des établissements d'enseignement et de recherche français ou étrangers, des laboratoires publics ou privés. 


\title{
REVUE DE PHYSIQUE APPLIQUÉE
}

\section{Accélérateurs et nouveaux concepts d'accélération de particules}

\author{
B. Heusch $\left({ }^{1}\right)$ et G. Matthieussent $\left({ }^{2}\right)$ \\ ( $\left.{ }^{1}\right)$ Centre de Recherches Nucléaires, Strasbourg, France \\ $\left({ }^{2}\right)$ CNRS, Laboratoire de Physique des Gaz et des Plasmas, Orsay, France
}

(Reçu le 15 octobre 1987, accepté le 28 juin 1988)

Les développements récents en matière d'accélérateurs de particules a été le thème d'un colloque qui s'est tenu à Strasbourg, en juillet 1987, lors de la conférence de la Société Française de Physique [1]. Ce numéro de la Revue Appliquée, sans être un compte rendu exhaustif de ce colloque, en est issu.

Une cinquantaine de physiciens, provenant de la physique nucléaire, de celle des particules élémentaires ainsi que de l'astrophysique et de la physique des plasmas ont pu faire le point sur les projets en cours et les nouveaux concepts d'accélération apparus ces dernières années. Outre l'information réciproque de physiciens d'origines différentes, ce colloque a confirmé qu'une fructueuse collaboration s'était déjà engagée autour des techniques d'accélération utilisant les plasmas et, plus précisément, autour des objectifs à atteindre quant à l'énergie et la luminosité des machines futures.

Pour explorer le domaine d'existence du boson de Higgs, la structure des leptons et des quarks et répondre à bien d'autres questions posées par la théorie [2], les physiciens des particules élémentaires envisagent la construction d'accélérateurs susceptibles de produire des particules dont l'énergie dépasse la dizaine de $\mathrm{TeV}$. Bien que durant les quarante dernières années l'énergie de ces machines a été multipliée par cinq ordres de grandeur, leur coût par $\mathrm{MeV}$ a été réduit sensiblement dans les mêmes proportions, et les machines à venir dans les dix ou vingt prochaines années ne seront que des extrapolations des accélérateurs en fonctionnement. Ceux-ci fournissent des champs accélérateurs de l'ordre de la dizaine de $\mathrm{Mev} / \mathrm{m}$ et les raffinements techniques (cavités supraconductrices, utilisation d'ondes millimétriques) ne permettront sans doute pas de dépasser la centaine de $\mathrm{MeV} / \mathrm{m}$.

Toutefois l'accroissement phénoménal de l'énergie des particules délivrées par les accélérateurs a été obtenu certes, par le développement de technologies existantes, mais surtout par l'émergence d'idées nouvelles.

De nouveaux concepts sont ainsi apparus ces dix dernières années, faisant appel aux plasmas $[3,4]$ et aux progrès récents réalisés dans la technologie des lasers de puissance [5] et des accélérateurs forts courants, moyenne tension [6]. Pourquoi des plasmas ? Dans les accélérateurs classiques, la limitation principale du champ électrique provient du claquage à la surface des matériaux ; l'utilisation d'un plasma, préalablement complètement ionisé, comme milieu support du champ électrique lève cette limitation. Des ondes longitudinales de plasma peuvent alors être excitées dans ce milieu de manière résonnante et avec des amplitudes de champ électrique de l'ordre de la dizaine de $\mathrm{GeV} / \mathrm{m}$ soit par le battement de deux ondes électromagnétiques issues de lasers de puissance [3] (quelques joules et quelques dizaines de picosecondes) soit par le sillage d'un faisceau d'électrons relativistes [4] (quelques $\mathrm{MeV}$ et quelques $100 \mathrm{kA}$ pendant des nanosecondes).

Ces nouveaux concepts d'accélérations en sont au stade des expériences de principe $[5,6]$ en laboratoire, sur des plasmas de longueur millimétrique et de densités voisines de $10^{17}$ particules par $\mathrm{cm}^{3}$; des champs électriques de l'ordre du $\mathrm{GV} / \mathrm{m}$ ont été obtenus par battement. Des expériences d'accélération d'électrons, avec des gains espérés de l'ordre de la dizaine de $\mathrm{MeV}$, sont en cours.

Pour atteindre des énergies de quelques dizaines de $\mathrm{TeV}$, un accélérateur basé sur l'un ou l'autre de ces concepts [7] devrait comporter de nombreux modules successifs; de nombreuses inconnues demeurent quant à la luminosité, la dispersion et le rendement en énergie, facteurs dépendant, entre autres, de la synchronisation des modules et de la réutilisation des faisceaux. Le but des expériences actuelles est donc, seulement, de démontrer la faisabilité du principe physique sur lequel pourrait 
s'appuyer une nouvelle génération d'accélérateurs de particules [8].

En physique nucléaire des découvertes importantes ont été possibles grâce aux réactions induites par des ions lourds sur les noyaux. En effet, par rapport à l'utilisation de faisceaux de particules légères, tout en conservant la sélectivité des mécanismes mis en jeu, les ions lourds permettent d'amener dans le système étudié un grand moment angulaire linéaire ce qui a permis d'étudier la matière nucléaire aux limites extrêmes de la stabilité. C'est ainsi que des résultats très intéressants ont été obtenus dans des domaines fondamentaux aussi divers que l'existence de nouveaux isotopes très loin de la stabilité, ou les limites de la fusion nucléaire et la fission induite observée pour pratiquement tous les noyaux, ou sur les formes des noyaux avec l'observation, par exemple, de superdéformations, ou encore la fragmentation nucléaire et les processus séquentiels [9].

L'étude des collisions entre noyaux atomiques entre 10 et $100 \mathrm{MeV} / \mathrm{n}$ a également permis d'étudier le passage de la prédominance du champ moyen nucléaire, à des énergies typiquement inférieures à $20 \mathrm{MeV} / \mathrm{n}$, au régime dominant de l'interaction nucléon nucléon au-delà de l'énergie de Fermi des nucléons dans les noyaux qui est aux environs de 35 $\mathrm{MeV} / \mathrm{n}$. Ces découvertes ont été rendues possible par des développements souvent récents, portant tant sur tous les types de sources d'ions que sur les accélérateurs, électrostatiques par exemple, qui couvrent le mieux le domaine basse énergie et celui des machines du type cyclo et synchroton utilisées aux plus grandes énergies citées ci-dessus.

Le tandem accélérateur de $35 \mathrm{MeV}$, VIVITRON, en cours de construction à Strasbourg débouche [10] sur une nouvelle génération de machines électrostatiques ; sa conception originale minimise les claquages en assurant une meilleure répartition de l'énergie électrique stockée. A partir de 1990, cette machine doit fournir pour les éléments légers, des ions multichargés avec une énergie de $20 \mathrm{MeV} / \mathrm{n}$ et pour les éléments lourds de $5 \mathrm{MeV} / \mathrm{n}$ avec des intensités de l'ordre de la centaine de nA.

Outre la prouesse technique de gérer des tensions aussi élevées que $35 \mathrm{MV}$, sa qualité essentielle est surtout liée aux qualités des faisceaux et à la facilité de changer aussi bien l'énergie que la nature de l'ion accéléré. Une telle machine peut éventuellement également servir d'injecteur pour des post-accélérateurs. De telles associations de machines sont déjà opérationnelles comme à Oak Ridge, Stony Brook, Chalk River ou Berlin, ou en cours de réalisation comme à Daresbury, Catane ou Saclay par exemple. Dans ce dernier cas [11] c'est un tandem de 9 MV qui fournit un faisceau au post-accélérateur constitué de cavités résonnantes supraconductrices utilisant un champ haute fréquence avec un gradient accélérateur de l'ordre de $2 \mathrm{MeV} / \mathrm{m}$; les énergies d'ions lourds y sont cependant limitées à environ 10 à $15 \mathrm{MeV} / \mathrm{n}$. Dans une association machine électrostatique cyclotron, soit à température ambiante (Berlin) ou supraconducteur (Chalk River, Catane), ces énergies maximales seront de l'ordre de $50 \mathrm{MeV} / \mathrm{n}$.

Pour des énergies de la dizaine à la centaine de $\mathrm{MeV} / \mathrm{n}$ des cyclotrons, voir des associations de cyclotrons, supraconducteurs (AGOR, MSU) ou non (SARA, GANIL) sont utilisés. Dans certains cas ce sont uniquement des associations de cavités résonnantes qui sont utilisées, là aussi, supraconductrices (MSU) ou non (GSI-Darmstadt). Enfin audelà de $100 \mathrm{MeV} / \mathrm{n}$ s'ouvre le domaine des synchrotrons tels SATURNE à Saclay $(1 \mathrm{GeV} / \mathrm{n})$ ou à Darmstadt (SIS, $2 \mathrm{GeV} / \mathrm{n}$ ) où l'adjonction d'un anneau de stockage est en cours de réalisation [12].

Au-delà de la physique nucléaire, une des applications futuristes des faisceaux d'ions lourds de forte énergie a été proposée en confinement inertiel [13] : la compression jusqu'à 1000 fois la densité du solide de microballons remplis de deuterium et de tritium pour $\mathrm{y}$ amorcer des réactions de fusion thermonucléaire. Les énergies d'ions lourds, par exemple du $\mathrm{Bi}^{+}$, à mettre en jeu sont de l'ordre de la $10 \mathrm{GeV}$ et les intensités de l'ordre de la dizaine de kA. Ces caractéristiques pourraient être obtenues par l'utilisation de combinaisons d'accélérateurs RF, de machines linéaires et d'anneaux de stockage. La comparaison entre les intensités de faisceau mises en jeu dans un tel projet et les intensités actuelles montre l'étendue des progrès technologiques à réaliser.

Les articles publiés dans ce numéro spécial recouvrent donc trois domaines: l'accélération conventionnelle, celle utilisant des plasmas et enfin les mécanismes d'accélération en astrophysique. Ceux réunis dans la première partie sont consacrés à l'état de l'art en matière d'électrons et d'ions : articles de synthèse sur l'ensemble des machines et techniques d'accélération, présentation du Vivitron et des différentes techniques utilisées. Dans une deuxième partie sont exposées les nouveaux concepts utilisant des plasmas et des faisceaux lasers. Les travaux expérimentaux entrepris au Canada (INRS-E) sur le battement d'ondes, et, à l'école Polytechnique, sur le Raman avant font l'objet de deux articles.

Les mécanismes d'accélération de particules en astrophysique ont fourni le troisième thème. Ils sont le plus souvent liés soit à la présence dans l'espace interstellaire d'ondes de choc quasiperpendiculaires, soit à une accélération de Fermi et entraînent l'apparition de particules jusqu'à une énergie de l'ordre du GeV.

\section{Remerciements.}

Les éditeurs associés expriment leurs remerciements aux auteurs et aux referés qui ont participé à l'élaboration de ce numéro spécial. 


\section{Bibliographie}

[1] Congrès SFP, Colloq, Accélérateurs et nouveaux concepts d'accélération de particules, Strasbourg 1987, Bull. SFP 65 (1987) 30.

[2] Le Courrier du CNRS, Dossier : Où va la physique des particules? (Nov; Dec. 1985) CNRS.

[3] Tajima, T., Dawson, J. M., Phys. Rev. Lett. 43 (1969) 267.

[4] Chen, P., Dawson, J. M., Huff, R. W., KatsouLEAS, T., Phys. Rev. Lett. 54 (1985) 693.

[5] Clayton, C. E., Joshi, C., Darrow, C., UmstaDER, D., Phys. Rev. Lett 54 (1985) 2343.

[6] Martin, F., Matte, J. P., Pepin, H., Ebrahim, N. E., IEEE Trans. Plasma Sci. PS-15 (1987) 167.

[7] IEEE Trans. Plasma Sci., Special issue on Plasma based high energy accelerators, PS-15 (1987) 2.
[8] Proc. of the ECFA Workshop on New developpements in Particule Acceleration technique (Orsay, France) 29. juin-4 juillet 1987, Ed. S. Turner.

[9] Lefort, M., J. Phys. Colloq. France (1986) C4-427 ; Proc. Ital. Phys. Soc., Eds. R. A. Ricci et C. Villi (1986) 467.

[10] Rapport d'activité 1987 VIVITRON, Centre de Recherches Nucléaires de Strasbourg, rapport CNR-VIV-50.

[11] Rapport d'activité 1986-1987 CEA, Département de Physique Nucléaire, rapport CEA-N-2550.

[12] KIENLE, P., rapport GSI-84-18 et rapport GSI-82-2.

[13] Deutsch, C., Ann. Phys. Fr. 11 (1986) 1. 